\title{
Influence of Radiation Dose, Photon Energy, and Reconstruction Kernel on rho/z Analysis in Spectral Computer Tomography: A Phantom Study
}

\author{
VASILIKI CHATZARAKI ${ }^{1,2}$, ALESSANDRA BOLSI $^{3}$, RAHEL A. KUBIK-HUCH $^{1}$, BERNHARD SCHMIDT $^{4}$, \\ ANTONY JOHN LOMAX ${ }^{3}$, DAMIEN C. WEBER ${ }^{3}$, MICHAEL THALI $^{2}$ and TILO NIEMANN ${ }^{1}$ \\ ${ }^{1}$ Department of Radiology, Kantonsspital Baden, Baden, Switzerland; \\ ${ }^{2}$ Institute of Forensic Medicine, University of Zurich, Zurich, Switzerland; \\ ${ }^{3}$ Paul Scherrer Institute (PSI), ETH Domain, Villigen, Switzerland; \\ ${ }^{4}$ Siemens Healthineers, Forchheim, Germany
}

\begin{abstract}
Background/Aim: The effective atomic number $\left(Z_{\text {eff }}\right)$ and electron density relative to water $\left(\varrho_{e}\right.$ or Rho) of elements can be derived in dual-energy computed tomography (DECT). The aim of this phantom study was to investigate the effect of different photon energies, radiation doses, and reconstruction kernels on $Z_{\text {eff }}$ and Rho measured in DECT. Materials and Methods: An anthropomorphic head phantom including five probes of known composition was scanned under three tube-voltage combinations in DECT: Sn140/100 kV, 140/80 kV and Sn140/80 kV with incremented radiation doses. Raw data were reconstructed with four reconstruction kernels (I30, I40, I50, and I70). Rho and $Z_{\text {eff }}$ were measured for each probe for all possible combinations of scan and reconstruction parameters. Results: DECT-based Rho and $Z_{\text {eff }}$ closely approached the reference values with a mean and maximum error of $1.7 \%$ and $6.8 \%$, respectively. Rho was lower for 140/80 kV compared with Sn140/100 kV and $5 n 140 / 80 \mathrm{kV}$ with differences being 0.009. $Z_{\text {eff }}$ differed among all tube voltages with the most prominent difference being 0.28 between $140 / 80 \mathrm{kV}$ and $S n 140 / 100 \mathrm{kV} . Z_{\text {eff }}$ was lower in I70 compared with those of 130 and I40 with a difference of 0.07. Varying radiation dose yielded a variation of 0.0002 in Rho and 0.03 in Z, both considered negligible in practice. Conclusion: DECT comprises a feasible method for the extraction of material-specific information. Slight
\end{abstract}

This article is freely accessible online.

Correspondence to: Tilo Niemann, Kantonsspital Baden, Im Ergel 1, CH-5404 Baden, Switzerland. Tel: +41 (0)564862111, e-mail: Tilo.Niemann@ksb.ch

Key Words: Tomography, $\mathrm{X}$-ray computed, radiation dosage, image reconstruction. variations should be taken into account when different radiation doses, photon energies, and kernels are applied; however, they are considered small and in practice not crucial for an effective tissue differentiation.

Traditional single-energy computed tomography (SECT) lacks high sensitivity in soft tissue differentiation because the attenuation numbers of many organs and tissues show overlaps in Hounsfield unit (HU) measurements. These overlaps arise because the linear coefficients of attenuation of the tissues are similar and mainly depend on two physical effects: photoelectric absorption and Compton scattering. The photoelectric absorption refers to the energy of X-ray photons that interact with the tightly bound electrons of the inner-shell, specifically, the K-shell. These interactions lead to the absorption of X-ray photons while electrons are ejected (1-3). The atomic number $\mathrm{Z}$ corresponds to the number of protons in the nucleus. As the K-shell binding energy is proportional to $\mathrm{Z}$ of an element, the photoelectric effect depends strongly on $\mathrm{Z}$ of the scanned material (approximately proportional to $Z^{3}$ ) (3). Photoelectric absorption depends on the incident photon energy (beam energy). The more the initial X-ray photon energy approaches the $\mathrm{K}$-shell binding energy or so called " $\mathrm{K}$ edge", the more probable the photoelectric effect is to occur (3). The photoelectric effect dominates at lower energies (4). Compton scattering refers to the ejection of the weakly bound outer shell electrons at different angles by the interaction with the X-ray photons. It is independent of photon energy at energies $>30 \mathrm{keV}$, but depends on the mass density of the scanned material (4) and dominates at low Z (1-3). Prominent Compton scattering leads to loss of image contrast $(3,5,6)$.

Dual-energy computed tomography (DECT) was first described in $1973(2,7)$. The objective, when used in clinical 


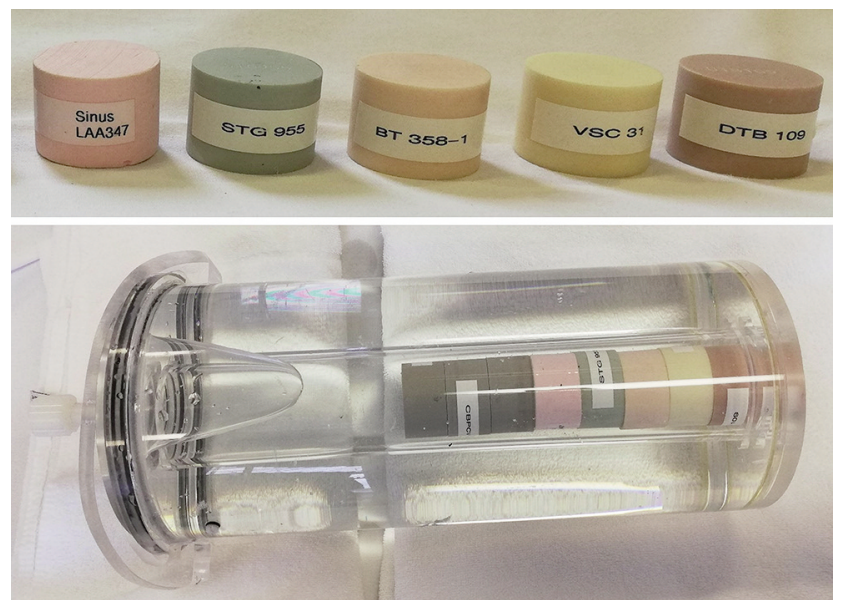

Figure 1. Anthropometric head phantom with $16 \mathrm{~cm}$ diameter filled with tap water and cylindrical probes (24).

practice, is to isolate and quantify different elements for tissue differentiation by simultaneously or consecutively scanning materials (depending on the computed-tomography (CT) scanner used, i.e., single or dual-source) in two different X-ray energy spectra (8). This concept allows the extraction of material-specific information based on the trends observed in the linear coefficients of attenuation within the different energy levels of the material. Differences that are not obvious by simple HU measurements on SECT may be revealed in the significant $\mathrm{Z}$ differences between different materials reflected in their coefficients (9). As the range between the applied radiation dose levels increases, the variations of coefficients of attenuation, and subsequently of their matrices, become more prominent and allow for a DECT-based tissue differentiation (9).

DECT has increasingly been applied during the past years for the elemental discrimination of urinary stones $(10,11)$, renal cell carcinoma (12), the differentiation of adrenal gland lesions (13), and the detection of uric acid crystals in patients with gout (14), among other medical conditions. Given the difference in $\mathrm{HU}(\Delta \mathrm{HU})$ for the same element at different energy levels, both the effective atomic number $\left(\mathrm{Z}_{\text {eff }}\right)$ and electron density relative to water $\left(\mathrm{Rho}\right.$ or $\left.\varrho_{e}\right)$ can be derived with small errors through DECT Rho/Z imaging data $(2,15-17)$.

Computationally intensive algorithms known as reconstruction kernels are used to modify the frequency content of the image data prior to back projection during image reconstruction in a CT scanner. Kernels adjust the spatial resolution and therefore affect the image quality by sharpening or softening the image. Different kernels exist for the evaluation of different anatomical structures, i.e., soft tissue and bone kernels. Evidence based on SECT have shown that the application of different reconstruction kernels affects attenuation measurements, especially of tissues with extreme low or high attenuation values taking as reference for the central $\mathrm{HU}$ regions, i.e., that of water with $0 \mathrm{HU}$ (1821). As the spatial frequency of a reconstruction kernel increases, i.e., as the kernel sharpens, edge-ringing artifacts appear and image noise increases leading to a broadened distribution of the attenuation values derived from the CTimage (18). Thus, softer kernels have generally been recommended for accurate attenuation measurements and for subsequent comparisons, for example, regarding the assessment of pulmonary emphysema (22). Hünemohr, et al. (23) observed a significant effect of image noise on $Z_{\text {eff }}$ and $\mathrm{Q}_{\mathrm{e}}$ values measured in DECT with a standard deviation (SD) of $10 \%$ in the mass fraction predictions of carbon and oxygen (23).

The aim of this phantom study was to investigate the effect of different combinations of photon energy (tube voltage), radiation dose levels (tube current), and reconstruction kernels on $Z_{\text {eff }}$ and Rho, the values of which are obtained in DECT Rho/Z imaging for tissue type differentiation.

\section{Materials and Methods}

Phantom scanning. An anthropometric head CT calibration phantom (CIRS, model 711HN modified) of 16-cm diameter filled with tap water and included five cylindrical probes of known composition was used (Figure 1). These probes were substitutes for sinus cavities, soft tissue, brain, spinal cord, and trabecular bone (Table I) (24). DECT of the phantom was performed in a dual-source CT SOMATOM Definition Flash scanner (Siemens Healthineers, Erlangen, Germany). The following scanning parameter settings involving three different voltage combinations were applied: 1) Sn140/100 kV, ref.mAs 20/40/60/80/100/120/140/160/180/200, rotation time $0.5 \mathrm{~s}$, pitch 1.0 , collimation $128 \times 0.6 \mathrm{~mm}$; 2) $140 / 80$ $\mathrm{kV}$, ref.mAs 20/40/60/80/100, rotation time $1 \mathrm{~s}$, pitch 1.0 , collimation $128 \times 0.6 \mathrm{~mm}$; 3) $\mathrm{Sn} 140 / 80 \mathrm{kV}$, ref.mAs $40 / 60 / 80 / 100 / 120 / 140 / 160 / 180 / 200$, rotation time $0.5 \mathrm{~s}$, pitch 1.0 , collimation $128 \times 0.6 \mathrm{~mm}$ (Sn denotes the use of a tin filter).

Post-processing - application of different reconstruction algorithms and Rho/Z measurements. Raw data obtained by all scans of the various voltage and current combinations of the tube were reconstructed in four different reconstruction kernels: I30 (medium smooth sharpness), I40 (medium sharpness), I50 (medium sharp), and $\mathrm{I} 70$ (very sharp). All Images were evaluated using Syngo.via ${ }^{\circledR}$ software for multimodality reading (Syngo.via Dual Energy, Siemens Healthcare GmbH 2009-2018, Version 05.01.000.0030, Erlangen, Germany).

One reader with four years of experience in cross-sectional imaging performed the attenuation measurements in the Rho/Z application profile in Syngo.via ${ }^{\circledR}$ (Siemens Healthineers, Forchheim, Germany). The electron density relative to water $\left(\mathrm{HU}_{\mathrm{Rho}}\right)$ and the effective atomic number $\left(\mathrm{Z}_{\mathrm{eff}}\right)$ were measured in this profile for each probe situated in the middle of the cylinder after axis correction (Figure 2). The reader was free to adjust the windowing for the most optimal margin differentiation for each of 
Table I. Properties of the cylindrical probes according to Albertini et al. (24), and the Rho and Z values measured on DECT and their extracted measurement errors.

\begin{tabular}{lccccccccc}
\hline Material & Substitute for & $\begin{array}{c}\text { Thickness } \\
(\mathrm{mm})\end{array}$ & $\begin{array}{c}\text { Density } \\
\left(\mathrm{g} / \mathrm{cm}^{3}\right)\end{array}$ & Rho & $\mathrm{Z}$ & Rho-DECT & Zeff-DECT & Rho-error & $\mathrm{Z}_{\mathrm{eff}}$-error \\
\hline DTB 109 & Trabecular bone & 25.52 & 1.16 & 1.116 & 10.05 & $1.151 \pm 0.008$ & $9.92 \pm 0.1$ & $0.035(3.1 \%)$ & $0.13(1.3 \%)$ \\
VSC 31 & Spinal cord & 25.26 & 1.07 & 1.033 & 7.43 & $1.037 \pm 0.005$ & $7.46 \pm 0.15$ & $0.004(0.4 \%)$ & $0.03(0.4 \%)$ \\
BT 358-1 & $\quad$ Brain & 25.48 & 1.07 & 1.040 & 7.44 & $1.039 \pm 0.002$ & $7.50 \pm 0.12$ & $0.001(0.1 \%)$ & $0.06(0.8 \%)$ \\
STG 955 & Soft tissue & 25.15 & 1.05 & 1.024 & 7.19 & $1.036 \pm 0.005$ & $7.26 \pm 0.16$ & $0.012(1.2 \%)$ & $0.07(0.9 \%)$ \\
LAA347 (LDT) & Sinus cavities & 25.67 & 0.21 & 0.204 & 7.16 & $0.19 \pm 0.001$ & - & $0.014(6.8 \%)$ & - \\
\hline
\end{tabular}

the probes. The size of the regions of interest used were adjusted as large as possible in the axial slices through each probe but excluded the rims.

The relative density to water is encoded in $\mathrm{HU}_{\mathrm{Rho}}$ in the $\mathrm{Rho} / \mathrm{Z}$ maps of Syngo.via, therefore a linear relation, was used to extract the electron density values as proposed by Saito et al. (16). For simplification, is written Rho in the following text.

Statistical analysis. Microsoft Excel 2016 (Microsoft Corp. Redmond, WA, USA) was used for recording attenuation measurements and creating the graphs for this study. R statistics package (25) was used in the statistical evaluation of the results. Rho and $\mathrm{Z}_{\text {eff }}$ values for each photon energy and reconstruction kernel combination were averaged over the different radiation doses (tube currents); moreover, DECT-based measurements were subtracted from the known reference values for each probe for the estimation of the measurement error. With photon energy and reconstruction kernel as fixed effects and the phantom probe and radiation dose as random effects, a repeated-measures linear-mixedeffect model was applied to explore the effect of photon energy and reconstruction kernel on Rho and $\mathrm{Z}_{\text {eff }}$ values, respectively. Pairwise comparisons with Tukey's Honest-Significant-Difference method were performed to compare the means among the distinct photon energy and reconstruction kernel groups. The level of significance was 0.05 .

\section{Results}

Rho and $\mathrm{Z}_{\text {eff }}$ values in the different photon-energy and reconstruction-kernel combinations were averaged over the different tube currents. Graphics of the Rho and $\mathrm{Z}_{\text {eff }}$ measurements of each probe are shown in Figure 3 and Figure 4, respectively.

Rho. Rho values measured in DECT after averaging all measurements over the different photon energies, radiation doses, and the reconstruction kernels that were applied are presented in Table I for each phantom probe. DECT-based Rho measurements closely approached the reference Rho values of each probe with the maximum measurement error being $0.014(6.8 \%)$ for the LAA347 probe.

The results from random effects showed that the estimated $\mathrm{SD}$ of the Rho values in relation to radiation dose (tube current) was $0.0002(95 \% \mathrm{CI}=0-0.0007)$.
From the results for the fixed effects, the photon energy (tube voltage) showed a significant effect on Rho values (repeated ANOVA: $p<0.001$, Table II). Rho values were significantly lower for $140 / 80 \mathrm{kV}$ compared with those for both $\mathrm{Sn} 140 / 100 \mathrm{kV}$ and $\mathrm{Sn} 140 / 80 \mathrm{kV}$, the Rho difference being 0.009 between $140 / 80 \mathrm{kV}$ and $\mathrm{Sn} 140 / 100 \mathrm{kV}$ and 0.008 between 140/80 and $\mathrm{Sn} 140 / 80 \mathrm{kV}$ (pairwise comparison with Tukey's method: both $p<0.001$, Table II). No significant differences were noted between Sn140/100 kV and Sn140/80 $\mathrm{kV}$ energies with the difference in Rho being notably lower (0.002) (pairwise comparison with Tukey's method: $p=0.3$ ).

There was no significant effect of the different reconstruction kernels on Rho values (repeated ANOVA: $p=0.99$, Table II).

$Z_{\text {eff. }}$ DECT-based $Z_{\text {eff }}$ measurements closely approached the reference $\mathrm{Z}$ values for each probe with the maximum measurement error being 0.13 (1.3\%) for the DTB109 probe (Table I).

The results of the random effects showed that the estimated $\mathrm{SD}$ of the $\mathrm{Z}$ values in relation to radiation dose (tube current) was $0.03(95 \% \mathrm{CI}=0.02-0.06)$.

Similarly, from the results on the fixed effects, both photon energy (tube voltage) and reconstruction kernel were found to have a significant effect on $\mathrm{Z}_{\text {eff }}$ values (repeated ANOVA for both radiation dose and reconstruction kernel: $p<0.001$, Table II):

The $\mathrm{Z}_{\text {eff }}$ values were significantly different between all three different photon energies (pairwise comparisons with Tukey's method for all three pairs: $p<0.001$ ), whereas the most prominent differences were found between $140 / 80 \mathrm{kV}$ and $\operatorname{Sn} 140 / 100 \mathrm{kV}(0.28)$ and $140 / 80 \mathrm{kV}$ and $\mathrm{Sn} 140 / 80 \mathrm{kV}$ (0.2). The $Z_{\text {eff }}$ difference between $\mathrm{Sn} 140 / 100 \mathrm{kV}$ and Sn140/80 kV was less prominent (0.09) but also statistically significant (Table II).

$\mathrm{Z}_{\text {eff }}$ values were significantly lower in I70 compared with those of the I30 and I40 kernels (Tukey's pairwise comparison: both pairs $p<0.001$ with both $\mathrm{Z}_{\text {eff }}$ differences being approximately 0.07 ). No further significant differences in $\mathrm{Z}_{\text {eff }}$ values were found between the other kernels (Tukey's pairwise comparisons: $p>0.05$, Table II). 


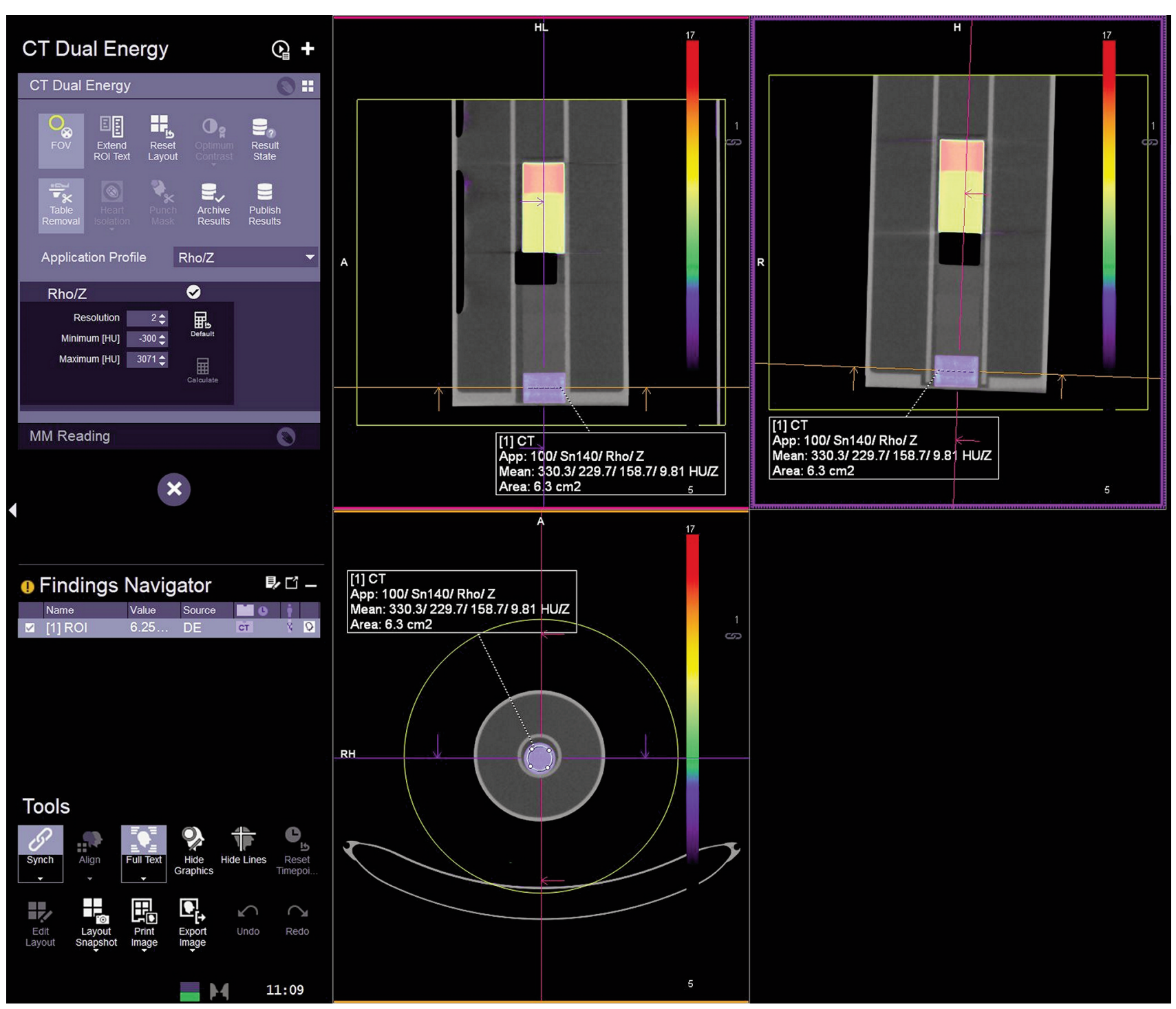

Figure 2. Syngo.via CT Dual energy «Rho/Z» application map: $H U_{R h o}$ and $Z_{\text {eff }}$ were measured for each probe in the middle of each cylinder after axis correction. Rho $\left(\varrho_{e}\right)$ was further calculated based on $H U_{R h o}$ as proposed by Saito et al. (16).

\section{Discussion}

To the best of our knowledge, this analysis is the first attempt to assess the possible effects of different radiation doses, photon energies, and reconstruction algorithms in the $\mathrm{DE}$ Rho/Z imaging measurements for material differentiation. This phantom study showed that the application of different radiation doses, photon energies, and reconstruction kernels might cause slight variations in Rho and $Z_{\text {eff }}$ measurements on DECT. Some differences in Rho and $Z_{\text {eff }}$ values among the different groups, although statistically significant, were small (maximum difference for Rho was 0.009 , for $Z_{\text {eff }} 0.28$, both between $S n 140 / 100$ and
$140 / 80 \mathrm{kV}$ tube voltages) and their practical relevance should be considered.

The Rho and $Z_{\text {eff }}$ values obtained by the DECTmeasurements closely reflected the reference $R$ ho and $Z_{\text {eff }}$ values provided by the manufacturer for each phantom probe (Table I). The measurement errors varied between $0.1 \%$ $6.8 \%$ for Rho and $0.4 \%-1.3 \%$ for $Z_{\text {eff. These percentages }}$ agree with previous studies in the literature presenting low measurement errors of $\mathrm{Rho} / \mathrm{Z}$ imaging $(2,16,17,26)$ indicating that DECT offers a feasible method for the extraction of material-specific information.

The photon energy as determined from the tube voltage was shown to have an effect on Rho/Z imaging. The 140/80 

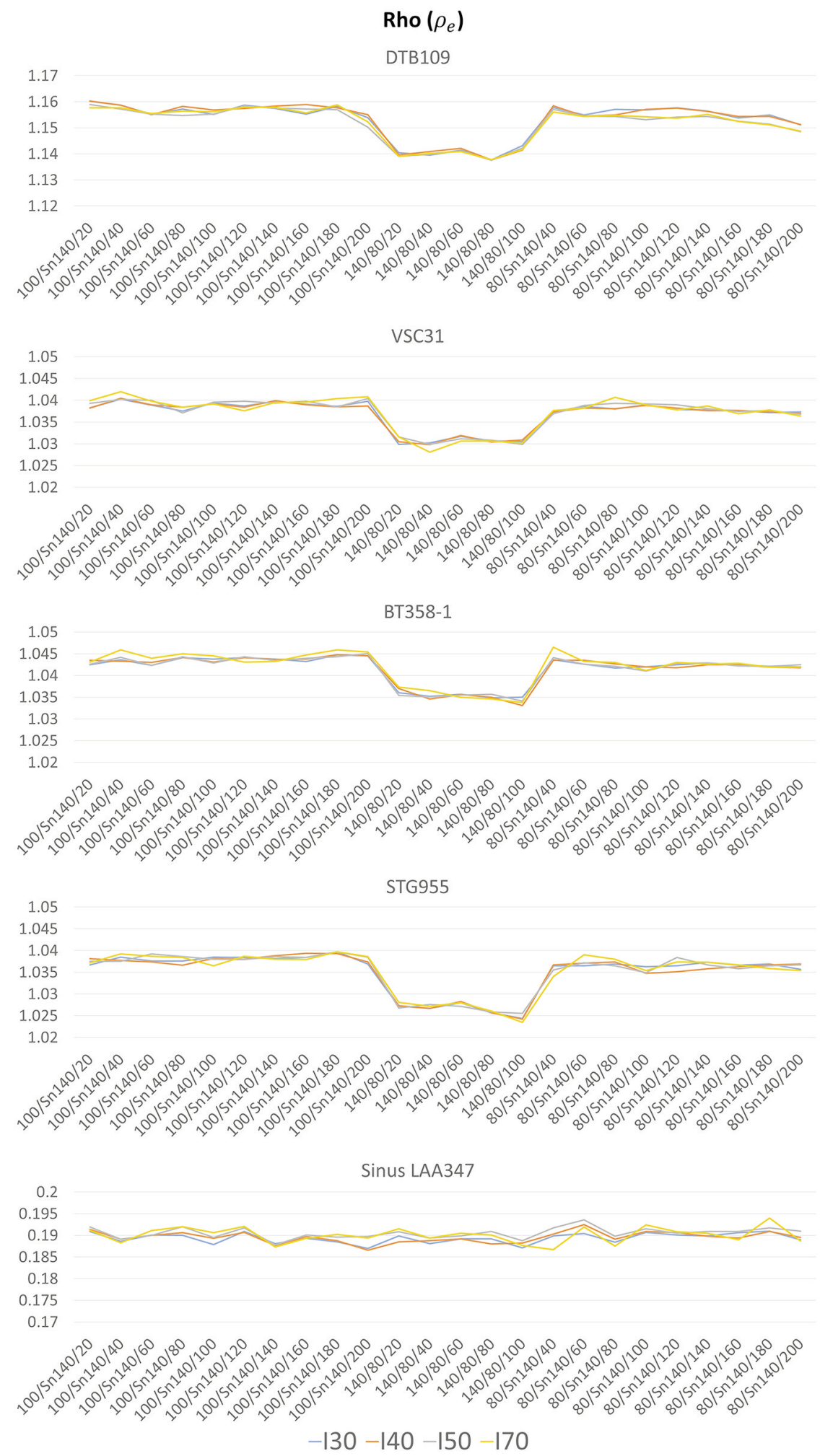

Figure 3. Graphs presenting the electron density relative to water Rho $\left(\varrho_{e}\right)$ measured in DE Rho/Z imaging [and calculated as proposed by Saito et al. (16)] for each probe at different photon energies (Sn140/100 kV, 140/80 kV, Sn140/80 kV) and radiation doses (20/40/../200 mAs) for the different reconstruction kernels $(130$, I40, I50, I70). SD arising from the different tube currents was in total 0.0002 for all phantom probes. The tube voltage had significant effect on Rho ( $p<0.001)$ : being significantly lower for 140/80 kV compared with both Sn140/100 kV and Sn140/80 kV with differences of 0.009 and 0.008 , respectively. The reconstruction kernels did not affect Rho $(p=0.99)$. 


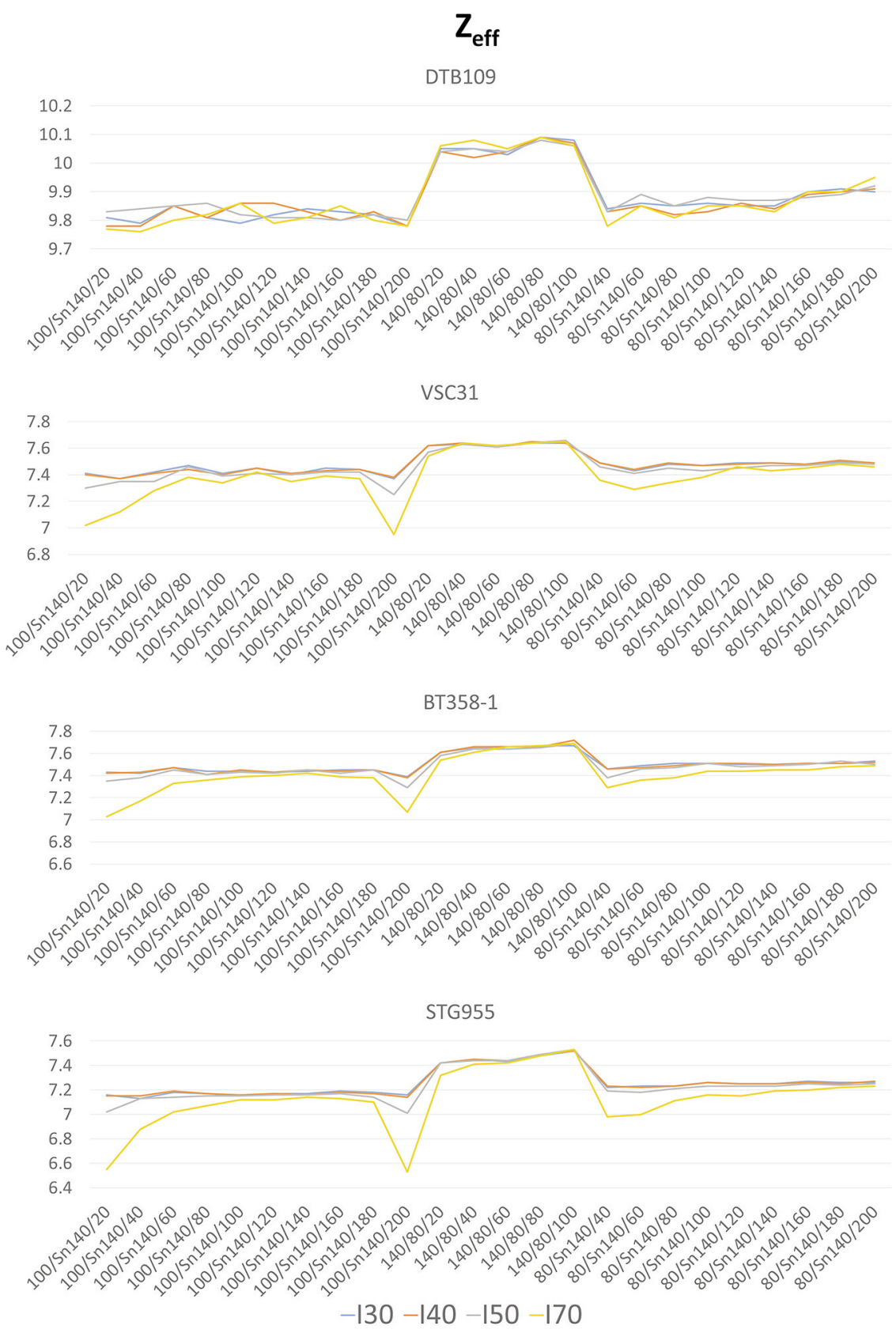

Figure 4. Graphs presenting the effective atomic number $\left(Z_{\text {eff }}\right)$ measured in DE Rho/Z imaging for each probe at different photon energies $($ Sn140/100 $\mathrm{kV}, 140 / 80 \mathrm{kV}, \mathrm{Sn} 140 / 80 \mathrm{kV})$ and radiation doses $(20 / 40 / . . / 200 \mathrm{mAs})$ within the different reconstruction kernels (I30, I40, I50, I70). The sinus $L A A 347$ probe results are not presented because $Z_{\text {eff }}$ values could not be extracted. $S D$ arising from the different tube currents was in total 0.03 . Both the tube voltage and reconstruction kernel had a significant effect on $Z_{\text {eff }}(p<0.001)$. $Z_{\text {eff }}$ was significantly different among all three voltages with the most prominent differences between $140 / 80 \mathrm{kV}$ and $S n 140 / 100 \mathrm{kV} . Z_{\text {eff }}$ was lower in I70 compared with those of I30 and I40.

$\mathrm{kV}$ combination primarily showed significantly lower Rho values compared with both $\mathrm{Sn} 140 / 100 \mathrm{kV}$ and $\mathrm{Sn} 140 / 80 \mathrm{kV}$. Spectral shaping for increased energy spectra separation is applied as an alternative to the standard low-dose protocols to reduce the radiation dose in constantly kept high tube voltages to achieve an adequate image quality with reduced image noise. This is attained using a tin filter placed between the standard aluminum filter and the patient to absorb the low-energy photons that are irrelevant in high-contrast imaging, for instance in that of bone or calcium urinary 
Table II. Mean absolute difference for Rho and $Z_{\text {eff }}$ between the different tube voltage groups and reconstruction kernels (averaged over the different tube currents).

\begin{tabular}{|c|c|c|}
\hline & \multicolumn{2}{|c|}{ Absolute difference $($ Mean \pm SD) } \\
\hline & Rho & $\mathrm{Z}_{\text {eff }}$ \\
\hline \multicolumn{3}{|l|}{ Tube voltage } \\
\hline Sn $140 / 100-140 / 80 \mathrm{kV}$ & $0.009 \pm 0.007^{* 1}$ & $0.28 \pm 0.09^{* 1}$ \\
\hline Sn140/100-Sn140/80 kV & $0.002 \pm 0.004^{* 2}$ & $0.09 \pm 0.08^{* 1}$ \\
\hline Sn140/80-140/80 kV & $0.008 \pm 0.006 * 1$ & $0.2 \pm 0.06 * 1$ \\
\hline \multicolumn{3}{|l|}{ Reconstruction kernel } \\
\hline $\mathrm{I} 30-\mathrm{I} 40$ & $0.0005 \pm 0.002 * 3$ & $0.01 \pm 0.01^{* 4}$ \\
\hline I30-I50 & $0.0008 \pm 0.003^{* 3}$ & $0.03 \pm 0.03^{* 4}$ \\
\hline $\mathrm{I} 30-\mathrm{I} 70$ & $0.0008 \pm 0.003 * 3$ & $0.07 \pm 0.12 * 1$ \\
\hline I40-I50 & $0.0006 \pm 0.003^{* 3}$ & $0.03 \pm 0.03^{* 4}$ \\
\hline $\mathrm{I} 40-\mathrm{I} 70$ & $0.001 \pm 0.003^{* 3}$ & $0.07 \pm 0.12 * 1$ \\
\hline $\mathrm{I} 50-\mathrm{I} 70$ & $0.0008 \pm 0.003^{* 3}$ & $0.05 \pm 0.09^{* 4}$ \\
\hline
\end{tabular}

${ }^{*}$ Pairwise comparison with Tukey's method: $p<0.001 .{ }^{* 2}$ Pairwise comparison with Tukey's method: $p=0.3$. ${ }^{*}$ Repeated measures ANOVA for Rho and reconstruction kernel: $p=0.99 .{ }^{*}$ Pairwise comparison with Tukey's method: $p>0.05$. Statistically significant differences are marked in bold.

stones $(27,28)$. With the tin filter, a decrease in radiationdose overlap of the two applied energy spectra occurs allowing for better tissue discrimination $(29,30)$. The tin filter is known to lower HU attenuation measurements and subsequently lower the contrast-to-noise ratio of an image, which is proportional to the HU attenuation of the structure under examination $(29,31)$. Rho is proportional to $\Delta H U$ between the two radiation spectra, (16). Taking into account that the radiation dose decreases when the tin filter is applied, the HU attenuation measurements (30) and, therefore, the $\Delta H U$ are expected to decrease as well, leading to lower Rho values as observed in this study.

The photon energy showed the exact opposite effect on $\mathrm{Z}_{\text {eff }}$ values compared with Rho, where the 140/80 kV combination was associated with higher $\mathrm{Z}_{\text {eff }}$ values compared with both $S n 140 / 100 \mathrm{kV}$ and $S n 140 / 80 \mathrm{kV}$. This may be explained by the fact that $Z_{\text {eff }}$ is inversely proportional to the HU attenuation and the Rho values according to the proposed algorithms for $\mathrm{Z}_{\text {eff }}$ calculation based on Rho on DECT $(16,32,33)$. These algorithms are commercially employed in the Rho/Z Maps application profile of the Syngo.via ${ }^{\circledR}$ software that was used for the measurements performed in this study. The $\mathrm{Z}_{\text {eff }}$ values could not be estimated in DECT for the LAA347 probe, the substitute for a sinus cavity mainly containing air, because of a known instability in the algorithm appearing in the $Q_{e} / \varrho_{e, w}$ component required for the calculation of $Z_{\text {eff }}$ (32).

The radiation dose as determined by the tube current was statistically handled as a random effect in the linear models used because of the small sample size within the distinct tube-current groups. This small size impedes a reliable statistical analysis for the extraction of a $p$-value regarding the effect of radiation dose on the Rho and $\mathrm{Z}_{\text {eff }}$. Therefore, a descriptive statistical approach was implemented using the calculation of the $\mathrm{SD}$ of Rho and $\mathrm{Z}_{\text {eff }}$ within each tube voltage group to reflect the variation incurred with the different tube currents applied. The SDs of both Rho (0.0002) and $\mathrm{Z}(0.03)$ are very small and therefore considered negligible from a practical point of view indicating that varying the radiation dose does not have a significant effect on Rho and $\mathrm{Z}_{\text {eff }}$ values critical in tissue and material differentiation based on DECT techniques.

The different reconstruction kernels did not significantly affect the Rho measurements in DECT. Regarding $\mathrm{Z}_{\text {eff }}$, the sharpest I70 was the only kernel found to be related with significantly lower values compared with the smoother I30 and I40 kernels. Sharper kernels provide a higher spatial resolution and image contrast at the expense of higher image noise and subsequently lower image quality (34), which may generate more prominent variations in the HU measurements on CT. Nevertheless, despite higher radiation doses being associated with better image quality, the most prominent variation in $\mathrm{Z}_{\text {eff }}$ in regard to $\mathrm{I} 70$ was unexplainably observed for $200 \mathrm{mAs}$ (Figure 4), even after repeated measurements were performed to exclude possible measurement errors. However, all differences reported to be statistically significant in this study were small (maximum differences: for Rho 0.009 , for $Z_{\text {eff }} 0.28$ ). In such instances, we can consider whether these small differences, although statistically significant, are effectively relevant and crucial in practice for material- and tissue-differentiation processes based on DECT. The highest significant difference for Rho was found to be $0.009 \pm 0.007$ and for $\mathrm{Z}_{\text {eff }} 0.28 \pm 0.09$, both between $\mathrm{Sn} 140 / 100 \mathrm{kV}$ and 140/80 kV photon energies. The presence of such small differences should always be considered in Rho and $\mathrm{Z}_{\text {eff }}$ evaluations when applying different tube voltages or reconstruction kernels; however, it is believed that for an effective tissue differentiation they may not be essential or misleading in practice.

The main limitation of this study is that it concerns a phantom study and current results may differ for other tissue types and human organs; this awaits further study. In addition, the X-ray tube of the CT-scanner used did not allow for a stepwise variation in the radiation dose between the range 100 to $200 \mathrm{mAs}$ in the $140 / 80 \mathrm{kV}$ combination (unlike in $\mathrm{Sn} 140 / 100 \mathrm{kV}$ and $\mathrm{Sn} 140 / 80 \mathrm{kV}$ ) because of dangers in overheating; therefore, a $140 / 80 \mathrm{kV}$ radiation dose could reach a maximum value of $100 \mathrm{mAs}$.

\section{Conclusion}

Varying radiation dose, photon energy, and reconstruction kernel may have an effect on Rho and $\mathrm{Z}_{\text {eff }}$ measurements in 
Rho/Z imaging applications performed in DECT for material and tissue differentiation. The application of a tin filter leads to a variation of approximately 0.009 in Rho and 0.28 in $\mathrm{Z}_{\text {eff }}$. The presence of these possible slight variations should be taken into account when evaluating Rho and $\mathrm{Z}_{\text {eff }}$ under different combinations of scan parameters and reconstruction algorithms that take place; however, the expected differences are considered small and in practice not critical for an effective DECT-based tissue differentiation.

\section{Conflicts of Interest}

Siemens Healthineers provided technical support for this study. One author is an employee of Siemens Healthineers (B.S.). He had no involvement in the study design; collection, analysis, and interpretation of data; writing of the report; or the decision to submit the article for publication. There is no further conflict of interest for any of the Authors.

\section{Authors' Contributions}

Study design and methodology (VC, AB, RK, BS, AL, DW, MT, $\mathrm{TN})$, design and construction of phantom ( $\mathrm{VC}, \mathrm{AB}, \mathrm{AL}, \mathrm{TN})$, image acquisition (VC, TN), Image analysis (VC, RK, TN), Data analysis (VC, RK, MT, TN), Writing the manuscript/statistics (VC, AB, RK, BS, AL, DW, MT, TN). All Authors have read and agreed to the published version of the manuscript.

\section{Acknowledgements}

V.C. has received a scientific grant from Guerbet, Zürich, Schweiz. The Authors thank Richard Haase, PhD, from Edanz Group (www.edanzediting.com/ac) for editing a draft of this manuscript.

\section{References}

1 Patino M, Prochowski A, Agrawal MD, Simeone FJ, Gupta R, Hahn PF and Sahani DV: Material separation using dual-energy CT: Current and emerging applications. Radiographics 36(4): 1087-1105, 2016. PMID: 27399237. DOI: 10.1148/rg. 2016150220

2 Goo HW and Goo JM: Dual-energy CT: New horizon in medical imaging. Korean J Radiol 18(4): 555-569, 2017. PMID: 28670151. DOI: $10.3348 / \mathrm{kjr} .2017 .18 .4 .555$

3 Marin D, Boll DT, Mileto A and Nelson RC: State of the art: dual-energy CT of the abdomen. Radiology 271(2): 327-342, 2014. PMID: 24761954. DOI: 10.1148/radiol.14131480

4 Fornaro J, Leschka S, Hibbeln D, Butler A, Anderson N, Pache G, Scheffel H, Wildermuth S, Alkadhi H and Stolzmann P: Dualand multi-energy CT: approach to functional imaging. Insights Imaging 2(2): 149-159, 2011. PMID: 22347944. DOI: 10.1007/ s13244-010-0057-0

5 Paziresh M, Kingston AM, Latham SJ, Fullagar WK and Myers GM: Tomography of atomic number and density of materials using dual-energy imaging and the Alvarez and Macovski attenuation model. J Appl Physics 119: 214901, 2016. DOI: $10.1063 / 1.4950807$
6 Rutherford RA, Pullan BR and Isherwood I: Measurement of effective atomic number and electron density using an EMI scanner. Neuroradiology 11(1): 15-21, 1976. PMID: 934468. DOI: $10.1007 / \mathrm{BF} 00327253$

7 Hounsfield GN: Computerized transverse axial scanning (tomography). 1. Description of system. Br J Radiol 46(552): 1016-1022, 1973. PMID: 4757352. DOI: 10.1259/0007-128546-552-1016

8 Petersilka M, Bruder H, Krauss B, Stierstorfer K and Flohr TG: Technical principles of dual source CT. Eur J Radiol 68(3): 362368, 2008. PMID: 18842371. DOI: 10.1016/j.ejrad.2008.08.013

9 Grupen $\mathrm{C}$ and Buvat I: Handbook of particle detection and imaging. Berlin, Heidelberg, Springer, pp. 885-890, 903-904, 2012. DOI: $10.1007 / 978-3-642-13271-1$

10 Franken A, Gevenois PA, Muylem AV, Howarth N and Keyzer $\mathrm{C}$ : In vivo differentiation of uric acid versus non-uric acid urinary calculi with third-generation dual-source dual-energy CT at reduced radiation dose. AJR Am J Roentgenol 210(2): 358363, 2018. PMID: 29166148. DOI: 10.2214/AJR.17.18091

11 Ananthakrishnan L, Duan X, Xi Y, Lewis MA, Pearle MS, Antonelli JA, Goerne H, Kolitz EM, Abbara S, Lenkinski RE, Fielding JR and Leyendecker JR: Dual-layer spectral detector $\mathrm{CT}$ : non-inferiority assessment compared to dual-source dualenergy CT in discriminating uric acid from non-uric acid renal stones ex vivo. Abdom Radiol (NY) 43(11): 3075-3081, 2018. PMID: 29626256. DOI: 10.1007/s00261-018-1589-x

12 Mileto A, Marin D, Alfaro-Cordoba M, Ramirez-Giraldo JC, Eusemann CD, Scribano E, Blandino A, Mazziotti S and Ascenti G: Iodine quantification to distinguish clear cell from papillary renal cell carcinoma at dual-energy multidetector CT: a multireader diagnostic performance study. Radiology 273(3): 813820, 2014. PMID: 25162309. DOI: 10.1148/radiol.14140171

13 Martin SS, Weidinger S, Czwikla R, Kaltenbach B, Albrecht $\mathrm{MH}$, Lenga L, Vogl TJ and Wichmann JL: Iodine and fat quantification for differentiation of adrenal gland adenomas from metastases using third-generation dual-source dual-energy computed tomography. Invest Radiol 53(3): 173-178, 2018. PMID: 28990974. DOI: 10.1097/RLI.0000000000000425

14 McLaughlin PD, Mallinson P, Lourenco P and Nicolaou S: Dualenergy computed tomography: advantages in the acute setting. Radiol Clin North Am 53(4): 619-38, vii, 2015. PMID: 26046502. DOI: $10.1016 /$ j.rcl.2015.02.016

15 Garcia LI, Azorin JF and Almansa JF: A new method to measure electron density and effective atomic number using dual-energy CT images. Phys Med Biol 61(1): 265-279, 2016. PMID: 26649484. DOI: $10.1088 / 0031-9155 / 61 / 1 / 265$

16 Saito $M$ and Sagara S: A simple formulation for deriving effective atomic numbers via electron density calibration from dual-energy CT data in the human body. Med Phys 44(6): 22932303, 2017. PMID: 28236659. DOI: 10.1002/mp.12176

17 Landry G, Reniers B, Granton PV, van Rooijen B, Beaulieu L, Wildberger JE and Verhaegen F: Extracting atomic numbers and electron densities from a dual source dual energy CT scanner: experiments and a simulation model. Radiother Oncol 100(3): 375379, 2011. PMID: 21924780. DOI: 10.1016/j.radonc.2011.08.029

18 Yuan R, Mayo JR, Hogg JC, Paré PD, McWilliams AM, Lam S and Coxson HO: The effects of radiation dose and CT manufacturer on measurements of lung densitometry. Chest 132(2): 617-623, 2007. PMID: 17573501. DOI: 10.1378/chest. $06-2325$ 
19 Rollano-Hijarrubia E, Stokking R, van der Meer F and Niessen WJ: Imaging of small high-density structures in CT A phantom study. Acad Radiol 13(7): 893-908, 2006. PMID: 16777564. DOI: $10.1016 /$ j.acra.2006.03.009

20 Gierada DS, Bierhals AJ, Choong CK, Bartel ST, Ritter JH, Das NA, Hong C, Pilgram TK, Bae KT, Whiting BR, Woods JC, Hogg JC, Lutey BA, Battafarano RJ, Cooper JD, Meyers BF and Patterson GA: Effects of CT section thickness and reconstruction kernel on emphysema quantification relationship to the magnitude of the CT emphysema index. Acad Radiol 17(2): 146156, 2010. PMID: 19931472. DOI: 10.1016/j.acra.2009.08.007

21 Sieren JP, Newell JD, Judy PF, Lynch DA, Chan KS, Guo J and Hoffman EA: Reference standard and statistical model for intersite and temporal comparisons of CT attenuation in a multicenter quantitative lung study. Med Phys 39(9): 5757-5767, 2012. PMID: 22957640. DOI: 10.1118/1.4747342

22 Rodriguez A, Ranallo FN, Judy PF and Fain SB: The effects of iterative reconstruction and kernel selection on quantitative computed tomography measures of lung density. Med Phys 44(6): 2267-2280, 2017. PMID: 28376262. DOI: 10.1002/ mp. 12255

23 Hünemohr N, Paganetti H, Greilich S, Jäkel O and Seco J: Tissue decomposition from dual energy CT data for MC based dose calculation in particle therapy. Med Phys 41(6): 061714, 2014. PMID: 24877809. DOI: 10.1118/1.4875976

24 Albertini F, Casiraghi M, Lorentini S, Rombi B and Lomax AJ: Experimental verification of IMPT treatment plans in an anthropomorphic phantom in the presence of delivery uncertainties. Phys Med Biol 56(14): 4415-4431, 2011. PMID: 21709345. DOI: $10.1088 / 0031-9155 / 56 / 14 / 012$

$25 \mathrm{R}$ Core Team: A language and environment for statistical computing. R Foundation for Statistical Computing, Vienna, Austria. 2020. Available at: https://www.R-project.org/ [Last accessed on January 28, 2021]

26 Bharati A, Mandal SR, Gupta AK, Seth A, Sharma R, Bhalla AS, Das CJ, Chatterjee S and Kumar P: Development of a method to determine electron density and effective atomic number of high atomic number solid materials using dual-energy computed tomography. J Med Phys 44(1): 49-56, 2019. PMID: 30983771. DOI: 10.4103/jmp.JMP_125_18

27 Petritsch B, Kosmala A, Weng AM and Bley TA: Tin-filtered $100 \mathrm{kV}$ ultra-low-dose $\mathrm{CT}$ of the paranasal sinus: Initial clinical results. PLoS One 14(5): e0216295, 2019. PMID: 31059550. DOI: 10.1371 /journal.pone. 0216295
28 Mozaffary A, Trabzonlu TA, Kim D and Yaghmai V: Comparison of tin filter-based spectral shaping CT and low-dose protocol for detection of urinary calculi. AJR Am J Roentgenol 212(4): 808-814, 2019. PMID: 30673337. DOI: 10.2214/AJR. 18.20154

29 Karlo C, Lauber A, Götti RP, Baumüller S, Stolzmann P, Scheffel H, Desbiolles L, Schmidt B, Marincek B, Alkadhi H and Leschka S: Dual-energy CT with tin filter technology for the discrimination of renal lesion proxies containing blood, protein, and contrast-agent. An experimental phantom study. Eur Radiol 21(2): 385-392, 2011. PMID: 20711733. DOI: $10.1007 / \mathrm{s} 00330-$ 010-1926-0

30 Primak AN, Giraldo JC, Eusemann CD, Schmidt B, Kantor B, Fletcher JG and McCollough CH: Dual-source dual-energy CT with additional tin filtration: Dose and image quality evaluation in phantoms and in vivo. AJR Am J Roentgenol 195(5): 11641174, 2010. PMID: 20966323. DOI: 10.2214/AJR.09.3956

31 Koonce JD, Vliegenthart R, Schoepf UJ, Schmidt B, Wahlquist AE, Nietert PJ, Bastarrika G, Flohr TG and Meinel FG: Accuracy of dual-energy computed tomography for the measurement of iodine concentration using cardiac CT protocols: validation in a phantom model. Eur Radiol 24(2): 512-518, 2014. PMID: 24141716. DOI: 10.1007/s00330-013-3040-6

32 Hünemohr N, Krauss B, Tremmel C, Ackermann B, Jäkel O and Greilich S: Experimental verification of ion stopping power prediction from dual energy CT data in tissue surrogates. Phys Med Biol 59(1): 83-96, 2014. PMID: 24334601. DOI: 10.1088/ 0031-9155/59/1/83

33 Möhler C, Wohlfahrt P, Richter C and Greilich S: Methodological accuracy of image-based electron density assessment using dualenergy computed tomography. Med Phys 44(6): 2429-2437, 2017. PMID: 28397977. DOI: $10.1002 / \mathrm{mp} .12265$

34 Shafiq-Ul-Hassan M, Zhang GG, Hunt DC, Latifi K, Ullah G, Gillies RJ and Moros EG: Accounting for reconstruction kernelinduced variability in $\mathrm{CT}$ radiomic features using noise power spectra. J Med Imaging (Bellingham) 5(1): 011013, 2018. PMID: 29285518. DOI: 10.1117/1.JMI.5.1.011013 